medRxiv preprint doi: https://doi.org/10.1101/2022.02.07.22270646; this version posted February 9, 2022. The copyright holder for this preprint (which was not certified by peer review) is the author/funder, who has granted medRxiv a license to display the preprint in perpetuity.

It is made available under a CC-BY-NC-ND 4.0 International license .

\title{
Post-acute sequelae of COVID-19 and adverse psychiatric outcomes: an etiology and risk systematic review protocol
}

\section{Andem Effiong ${ }^{1}$}

1. Faculty of Medicine, Memorial University of Newfoundland, St. John's, Newfoundland and Labrador, Canada

Correspondence to: Andem Effiong; andem.effiong@me.com

\begin{abstract}
Introduction The post-acute sequelae of COVID-19 (PASC) is a syndrome characterized by persistent COVID-19 symptoms or the onset of new symptoms following recovery from the initial or acute phase of the illness. Such symptoms often occur four or more weeks after being diagnosed with COVID-19. Although a lot of work has gone into understanding the long-term mental health effects of PASC, many questions related to the etiology and risk of this condition remain. Thus, this protocol is for a systematic review assessing the association between PASC and adverse psychiatric outcomes and whether people with PASC are at greater risk of developing an adverse psychiatric outcome than those without PASC.
\end{abstract}

Methods and analysis Various medical databases (e.g., PubMed and EMBASE) will be searched for eligible articles using predefined search criteria. Gray literature will also be explored. Epidemiological observational studies and secondary analyses of randomized controlled trials that report a quantitative relationship between PASC and at least one adverse psychiatric outcome will be included. The Population, Exposure of interest, Comparator, and Outcome (PECO) framework will be used as a standardized framework for the inclusion criteria. The Joanna Briggs Institute (JBI) critical appraisal tools will be used to assess methodological quality and critically appraise the risk of bias in included studies. A random-effects metaanalysis will be conducted if possible. A narrative synthesis will be performed if a meta-analysis is impossible due to substantial heterogeneity across studies. The Grading of Recommendations Assessment, Development and Evaluation (GRADE) approach will be used to rate the cumulative certainty of the evidence for all outcomes.

Ethics and Dissemination Ethical approval is not required for this study. The study results will be published in a peer-reviewed journal.

Prospero registration number CRD99999999999

Strengths and limitations of this study

- This study documents and addresses etiology, risk factors, and long-term symptoms of COVID-19 among people with post-acute sequelae of COVID-19 (PASC).

- It focuses on a key priority area for new evidence syntheses on the clinical management of COVID-19 and pandemic-related conditions. 
medRxiv preprint doi: https://doi.org/10.1101/2022.02.07.22270646; this version posted February 9, 2022. The copyright holder for this preprint (which was not certified by peer review) is the author/funder, who has granted medRxiv a license to display the preprint in perpetuity.

It is made available under a CC-BY-NC-ND 4.0 International license .

- It will include evidence on non-hospitalized and hospitalized patients with a history of PASC.

- Substantial heterogeneity across studies may limit the ability to perform a meta-analysis.

- Findings will inform disease prevention, decision-making, healthcare policy, and clinical research.

\section{BACKGROUND}

\section{Rationale}

Coronavirus disease 2019 (COVID-19) is a contagious illness caused by the severe acute respiratory syndrome coronavirus 2 (SARS-CoV-2). Persistent and long-lasting (>4 weeks ) symptoms following infection with acute COVID-19 have given rise to a syndrome known as post-acute sequelae of COVID-19 (PASC) or long COVID. ${ }^{1}$ Incidence and prevalence estimates for people with COVID-19 presenting with or reporting persistent psychiatric symptoms months following initial infection range from $0.8-49 \%{ }^{1,2,3,4,5}$ Among 44759 people with no recorded history of psychiatric illness, the estimated overall probability of being diagnosed with newonset psychiatric illness in the 90 days following a confirmed diagnosis of COVID-19 was 5.8\% in a retrospective cohort study. ${ }^{6}$ Similarly, clinical anxiety and depression, as well as other psychiatric sequelae, have been reported following diagnosis with COVID-19 in other studies. ${ }^{6,7}$, ${ }^{8}$ Although sex and age are considered to be sociodemographic risk factors for PASC, there is no consensus on other baseline clinical features that act as independent predictors of PASC. ${ }^{9,} 10$ The prevalence of PASC symptoms is higher in women compared with men. ${ }^{10}$ Among people aged $35-49$ years, the prevalence of PASC is $26.8 \%$ compared with $26.1 \%$ and $18 \%$ among people aged 50-69 years and 70 years or older, respectively. ${ }^{10}$

Persistent symptoms occur weeks and months after infection irrespective of initial disease severity (mild, moderate, severe, critical). ${ }^{11,12}$ Mendez et al. reported in their cross-sectional study that two months after discharge, neurocognitive impairment, psychiatric morbidity, and poor quality of life were markedly prevalent among 179 COVID-19 survivors who had been hospitalized. ${ }^{12}$ Nevertheless, Vannorsdall and Oh posit that current research on the post-acute phase following hospitalization has been conflicting due to the absence of a detailed, standardized neuropsychological evaluation of COVID-19 patients after hospitalization. ${ }^{13}$ In addition, they stated that literature on PASC and adverse mental health outcomes are mostly limited to studies that cannot establish causal relationships or lack generalizability (e.g., case reports, case series, and data obtained from cognitive screening instruments). ${ }^{13}$ Thus, more highquality studies are warranted. ${ }^{13}$

In a study where the short-term and long-term sequelae of COVID-19 were systematically evaluated, PASC was categorized as short-term (1 month), intermediate-term (2-5 months), and long-term ( $\geq 6$ months) following COVID-19 diagnosis. ${ }^{14}$ Clinical manifestations of PASC were classified into organ systems, i.e., cardiovascular, dermatologic, digestive, ear, nose, and throat; 
mental health, neurologic, and respiratory; constitutional symptoms; and functional mobility. ${ }^{14}$ The mechanisms leading to the post-acute and chronic neuropsychiatric manifestations of COVID-19 are thought to be due to the direct effect of the viral infection and the indirect effect on mental health due to social isolation, posttraumatic stress, and job loss. Specifically, correlations have been observed between COVID-19 posttraumatic stress scores, general distress, and sleep disruption. ${ }^{14,15}$ Despite those correlations, Khubchandani et al. stated that the causal pathways and etiology of adverse mental health outcomes in people who were infected with COVID-19 are multidimensional and complex. ${ }^{16}$

To clarify whether COVID-19 is a risk factor for psychiatric disorders and vice-versa, an electronic health record network cohort study of 69 million people consisting of 62354 people with a COVID-19 diagnosis compared the rates of psychiatric sequelae of health in the initial four months of the pandemic (January - April 2020) and subsequently (after April 2020). ${ }^{17 .}$ The study found that the rate of all diagnoses of psychiatric disorders and relapses was greater following a COVID-19 infection compared with after control health events (e.g., influenza infection, skin infection, other respiratory tract infections, and fracture). ${ }^{17}$ Similarly, a diagnosis of psychiatric disorder in the 12 months preceding the COVID-19 pandemic was associated with a $65 \%$ increased risk of COVID-19 (relative risk $(\mathrm{RR})=1.65,95 \%$ confidence interval $(\mathrm{CI})$ : 1.59-1.71; $\mathrm{p}<0.0001$ ) compared with a matched cohort of people with specific physical risk factors for COVID-19 minus a psychiatric diagnosis. ${ }^{17}$ Whereas these associations were partly attributed to illness severity and pandemic-related contextual factors (e.g., social isolation, overwhelmed healthcare systems, and stigma), they do not adequately account for observed differences in psychiatric sequelae. ${ }^{17}$ Moreover, the inability to conclusively determine why there were between twofold and threefold increases in the risk of neurologic and psychiatric complications following a COVID-19 infection, in this and other studies, calls for further examination of the association between COVID-19 and risk factors for psychiatric morbidity. $17,18,19$

With many long-term adverse mental health outcomes linked to COVID-19, effective interventions which optimize recovery and minimize relapse are needed. Such interventions may serve as appropriate tools to evaluate risk factors that may cause maladaptive psychiatric responses. ${ }^{20}$ Furthermore, they may aid with the management of anxiety, fear, frustration, stigma, and paranoia by mitigating psychopathological symptoms and reducing contextual stress. ${ }^{20}$ Interventions that have been assessed in COVID-19 patients include: virtual and physical psychotherapeutic approaches, e.g., cognitive behavioral therapy (CBT), emotional freedom techniques (EFT), and ultra-brief psychological interventions (UBPI); combined psychiatric and psychological interventions; technology and media; complementary and alternative therapies; self-care; spirituality and religion; and pharmacological. ${ }^{21,22}$

In a randomized controlled clinical trial of 51 people with COVID-19 consisting of an experimental group receiving progressive muscle relaxation (PMR) technology for 30 minutes each day for five consecutive days and a control group receiving only usual care and treatment, subjects in the experimental group reported lower depressive symptoms, lower anxiety levels, and better sleep quality compared with those in the control group. ${ }^{23}$ Another randomized control 
trial of 30 hospitalized patients with COVID-19 assigned to an experimental or control group reported an improvement in all outcome measures among intervention group subjects compared to controls. ${ }^{24}$ In that study, a short four-session crisis intervention package tailored to cover COVID-19-specific guidance was delivered by clinical psychologists. ${ }^{24}$ Topics covered included tension reduction, relaxation, adjustment, responsibility skills enhancement, and promoting resilience. ${ }^{24}$ Outcomes measures in the study were derived from the Depression, Anxiety, and Stress Scale (DASS21), Symptom Checklist 25 (SCL-25), and the abbreviated version of the World Health Organization Quality of Life assessment (WHOQOL-BREF). ${ }^{24}$ Lack of cultural specificity, methodological issues, small sample sizes, lack of follow-up, unadjusted confounding factors, and brief time spans in both studies limit their generalizability. ${ }^{23,24}$

During the COVID-19 pandemic, digital interventions to deliver health care have gained widespread acceptance. ${ }^{25}$ Remote care coordination and provision have been adopted to help reduce the risk of disease transmission. ${ }^{25}$ Mobile applications have also been used for contact tracing and information dissemination. ${ }^{25}$ Although an evidence synthesis of digital interventions to attenuate the adverse effects of the COVID-19 pandemic on public mental health highlighted their importance in mental disorder prevention and mental health promotion; it noted that evidence on their cost-effectiveness, process quality, and long-term outcomes is sparse. ${ }^{26}$ Furthermore, the negative impact and risks of the COVID-19 pandemic are sometimes more significant in vulnerable and clinically extremely vulnerable populations (e.g., people over the age of 70, pediatric patients with cystic fibrosis, or people with developmental disabilities) who may be digitally disadvantaged. ${ }^{26,27,28}$

Presently, it is unclear what duration of PASC, etiologies and risk factors are most associated with the manifestation or persistence of adverse psychiatric outcomes (e.g., depression, anxiety, substance use disorder, posttraumatic stress disorder, psychosis, dementia, suicide) compared with other health events. A prospective cohort study of patient-reported outcome measures (PROM) three months after initial COVID-19 symptom onset noted impairment with self-care and anxiety or depression as being present in $13 \%$ and $22 \%$ of its 78 subjects with at least one Charlson comorbidity at baseline compared to subjects without any Charlson comorbidities (4\% and $9 \%$ respectively). Among subjects without any Charlson comorbidities, $70 \%$ reported an abnormal PROM, and 33\% had at least one moderate issue in at least one EuroQol- 5 Dimension (EQ-5D). ${ }^{29}$ In addition, questions remain about the long-term ( $\geq 6$ months) outcomes of COVID-19. ${ }^{30}$

Although some studies indicate that most people who acquire COVID-19 are at risk of psychiatric sequelae and their symptoms tend to improve over time, others suggest that symptoms may worsen over time or point to a different disease trajectory. ${ }^{30,31}$ Research and any future recommendations about PASC and mental health should be guided by the best available evidence.

An initial search of peer-reviewed and gray literature found no systematic reviews and metaanalyses on the topic. This protocol is for a systematic review that assesses the literature on PASC duration and risk factors that act as determinants (etiologies) of adverse psychiatric outcomes. 


\section{Objectives}

The primary objective of this systematic review is to determine whether people with PASC are at greater risk of developing an adverse psychiatric outcome (depression, anxiety, substance use disorder, posttraumatic stress disorder, psychosis, dementia, suicide) than those without PASC.

Secondary review questions include the following:

- Does the association between PASC and an adverse psychiatric outcome vary with age, sex, the severity of COVID-19 (mild, moderate, severe, critical), and duration of PASC (short-term (1 month), intermediate-term (2-5 months), and long-term ( $\geq 6$ months) following COVID-19 diagnosis or hospital discharge)?

- Is PASC an independent risk factor for an adverse psychiatric outcome?

\section{METHODS}

This protocol has been drafted following the Preferred Reporting Items for Systematic Reviews and Meta-Analyses (PRISMA) guidance for protocols (PRISMA-P). ${ }^{32}$ The systematic review will explicitly report any amendments and modifications made to this protocol.

\section{Eligibility criteria}

Study design/characteristics: The review will include observational studies, namely, retrospective and prospective longitudinal cohort, case-control, cross-sectional, case series, and case reports. Secondary analyses of randomized controlled trials will also be included. Effect measures of risk factors, e.g., risk difference, relative risk, odds ratio, and hazard ratio central to the primary outcome, will be included. Risk factors predispose people with PASC to an adverse psychiatric outcome. Such risk factors are associated with an increased probability of people with PASC having a negative mental health outcome. Information on the relationship between risk factors and incidence of primary and secondary outcome measures will be included. Studies that do not report a quantitative relationship between PASC and at least one adverse psychiatric outcome will be excluded.

COVID-19 diagnosis must have been confirmed through clinical suspicion or with a positive nucleic acid amplification test (NAAT), e.g., reverse transcriptase-polymerase chain reaction (RT-PCR); antigen test; or serologic test (e.g., rapid serology test (RST) or enzyme-linked immunosorbent assay (ELISA)). ${ }^{33}$ Studies will be included if subjects were longitudinally observed since the initial diagnosis of COVID-19, i.e., during the acute phase or since the time of PASC onset (post-acute or chronic phase). A follow-up time of at least one month since the COVID-19 diagnosis is required. Primary and secondary outcomes will encompass etiology, risk factors, symptom and illness severity, duration of PASC, and adverse events.

Participants: Studies with adults as subjects (18 years or older) will be included. Pediatric and animal studies will not be included. There will be no sex, ethnicity, or race limitations. The 
search dates will range from December 2019 (date of first confirmed case of COVID-19) until March 2022 (the anticipated completion date of the review). COVID-19 filters will be used - if necessary - to limit search results to COVID-19 and PASC related articles.

Exposure:

Primary measure

- Post-acute sequelae of COVID-19 (PASC), for this review defined as a continuing symptomatic illness or the emergence of new symptomatic illness in people with a confirmed history of COVID-19 after recovery from the acute phase of illness. PASC will be categorized as short-term (1 month), intermediate-term (2-5 months), and longterm ( $\geq 6$ months) following COVID-19 diagnosis or hospital discharge.

Secondary measures

- Severity of COVID-19 (mild (including asymptomatic), moderate, severe, critical)

\section{Comparators(controls):}

Primary measure

- People with a confirmed history of COVID-19 without PASC

Secondary measures

- Severity of COVID-19 (mild (including asymptomatic), moderate, severe, critical)

\section{Outcomes:}

Primary outcome variable

Adverse psychiatric outcome

- Depression

- Anxiety

- Substance use disorder

- Posttraumatic stress disorder

- Psychosis

Secondary outcome variable

- Self-harm

- Suicide

\section{Information (evidence) sources and search strategies}

Information, including titles and abstracts extracted from evidence sources, will be initially screened against the review questions. Information deemed eligible for inclusion will undergo 
medRxiv preprint doi: https://doi.org/10.1101/2022.02.07.22270646; this version posted February 9, 2022. The copyright holder for this preprint (which was not certified by peer review) is the author/funder, who has granted medRxiv a license to display the preprint in perpetuity.

It is made available under a CC-BY-NC-ND 4.0 International license .

more comprehensive screening. Once an article, study, or review is considered suitable for inclusion, it will be placed in the list of included studies. The steps above will be done for each information source, after which duplicates will be removed. The study selection process will be described in a PRISMA flow diagram and reported in the systematic review.

AE will develop the search strategy in consultation with a medical research librarian. The following databases and evidence sources will be searched: PubMed, Ovid MEDLINE, EMBASE, JBI EBP Database, CINAHL Plus, UpToDate, APA PsycInfo, Google Scholar, ProQuest Dissertations \& Theses Global, Scopus, Web of Science, the University of Toronto COVID-19 Data \& Statistical Sources, Centre for Addiction and Mental Health (CAMH) COVID-19 National Survey Dashboard reports, and COVID-END. Gray literature will also be considered where appropriate. Search strategies will be comprehensive and adapted for each information source. See Appendix 1 for a sample of the PubMed search strategy.

The Covidence (Covidence, Melbourne, Australia) or JBI SUMARI software will be used during the systematic review process for screening, appraisal of evidence sources, data extraction, synthesis, and study completion.

\section{Study selection}

Information, including titles and abstracts, extracted from information sources will be initially screened by $\mathrm{AE}$ and a second reviewer against the research questions. Information deemed eligible for inclusion will undergo more comprehensive screening. Once an article, study, or review is considered suitable for inclusion, it will be placed in the list of included studies. The steps above will be done for each information source, after which duplicates will be removed. Disagreements on inclusion will be resolved through discussion or arbitration. The study selection process will be described in a PRISMA flow diagram and reported in the systematic review.

\section{Data extraction and management}

Data will be extracted on primary and secondary outcome measures following the PRISMA guideline for systematic reviews. ${ }^{35}$ Outcome and effect size measures (e.g., adjusted and unadjusted odds, risk ratios, hazard ratios, standard errors), p-values, and associated 95\% confidence intervals. RR for subgroups (e.g., age, sex, duration of PASC, COVID-19 severity) will be extracted if reported. The following data will also be extracted: authorship, publication year, journal name, study design, study location, sample size, baseline characteristics of subjects, demographics (age, sex, ethnicity, or race of subjects,'), study population characteristics (e.g., general population, prisoners, healthcare workers), the definition of PASC, duration of PASC, comorbidities, other risk factors, duration of follow-up, list of adjusted and unadjusted colliders (e.g., hospitalization, occupation, symptom recognition) and list of adjusted and unadjusted confounders (e.g., age, sex, nature of exposure, type of intervention), propensity methods. ${ }^{34}$ Two reviewers will conduct data extraction. Discrepancies in data extraction will be resolved through discussion or arbitration.

\section{Risk of bias in individual studies}


medRxiv preprint doi: https://doi.org/10.1101/2022.02.07.22270646; this version posted February 9, 2022. The copyright holder for this preprint (which was not certified by peer review) is the author/funder, who has granted medRxiv a license to display the preprint in perpetuity.

It is made available under a CC-BY-NC-ND 4.0 International license .

The JBI critical appraisal checklist will be used to determine the methodological quality and critically appraise the risk of bias for included studies. Information related to a variable (exposure, outcome, covariate), misclassification, confounding, participant selection, reverse causation, missing data, study power, and generalizability will be appraised. Two reviewers will initially pilot the checklist to enhance consistency, mitigate potential issues with mechanistic scoring, and mitigate performance bias in the overall risk of bias assessment. Studies that do not adequately report on statistical analyses or address confounding, biases (selection, performance, detection, attrition), and other biases will be deemed lower quality studies, i.e., when they consistently have 'no,' 'unclear, and 'not applicable' ratings' across relevant items.

\section{Data synthesis}

Summary treatment effects estimated as continuous outcomes will be converted to OR, RR, risk difference, and number needed-to-treat (NNT) with a 95\% confidence interval (plus the baseline risk) for easier interpretation where possible. A random-effects meta-analysis will be conducted if possible. Statistical heterogeneity across studies will be explored using Higgins $I^{2}$ and Cochran's Q statistics. A Cochran's $Q$ test based on a $\chi 2$ statistic with a $p<0.05$ and greater than the degrees of freedom (df) will indicate heterogeneity. The $I^{2}$ statistic will be interpreted as follows: $0-40 \%=$ minimal heterogeneity; $30-60 \%=$ moderate heterogeneity; $50-90 \%=$ substantial heterogeneity; $75-100 \%=$ considerable heterogeneity. If there is substantial heterogeneity, subgroup analysis (based on the duration of PASC or COVID-19 severity) will be conducted. Subgroup effect sizes (Cohen's $d$ or Hedges $g$ ) and correlations will be assessed and compared with unadjusted values to interpret for meaningful effects. Observed effects will be considered robust if the effect estimates of the primary outcome remain consistent or there are no large differences in the magnitude of effect across subgroups. Subgroup analyses will not be performed if there is minimal or moderate heterogeneity. A narrative synthesis will be performed if meta-analysis is not possible. The reasons for not pooling data (e.g., high statistical, methodological, and clinical heterogeneity) will be reported in the review. A methodological quality-based sensitivity analysis presented as a summary table will be used to assess the robustness of the findings. Authors of included studies with missing information will be contacted for clarification. The Grading of Recommendations, Assessment, Development, and Evaluations (GRADE) approach will be used to rate the overall certainty of the evidence obtained from the study.

\section{Patient and public involvement}

Input on the review questions and outcomes was informally sought from patients and people who had been previously diagnosed with COVID-19 and PASC.

\section{Ethics and dissemination}

Ethical approval is not required for this study. Study findings will be disseminated via preprints, peer-reviewed publications, conference abstracts, posters, plain language summaries, presentations, and infographics.

\section{Ethics statements}


medRxiv preprint doi: https://doi.org/10.1101/2022.02.07.22270646; this version posted February 9, 2022. The copyright holder for this preprint (which was not certified by peer review) is the author/funder, who has granted medRxiv a license to display the preprint in perpetuity.

\section{Patient consent for publication}

Not applicable.

Contributors: AE conceived, designed, and drafted the study protocol.

Funding: This work was supported by the Canadian Institute of Health Research (Grant number: NFRFR-2019-00012 ). The funding body will not have any role in the systematic review (and meta-analysis) process.

Competing interests: None declared.

Provenance and peer review: Not commissioned; externally peer-reviewed.

\section{REFERENCES}

1. Nalbandian A, Sehgal K, Gupta A, Madhavan MV, McGroder C, Stevens JS, Cook JR, Nordvig AS, Shalev D, Sehrawat TS, Ahluwalia N. Post-acute COVID-19 syndrome. Nature medicine. 2021 Apr;27(4):601-15.

2. Postolache TT, Benros ME, Brenner LA. Targetable biological mechanisms implicated in emergent psychiatric conditions associated with SARS-CoV-2 infection. JAMA psychiatry. 2021 Apr 1;78(4):353-4.

3. Taquet M, Geddes JR, Husain M, Luciano S, Harrison PJ. 6-month neurological and psychiatric outcomes in 236379 survivors of COVID-19: a retrospective cohort study using electronic health records. The Lancet Psychiatry. 2021 May 1;8(5):416-27.

4. Santomauro DF, Herrera AM, Shadid J, Zheng P, Ashbaugh C, Pigott DM, Abbafati C, Adolph C, Amlag JO, Aravkin AY, Bang-Jensen BL. Global prevalence and burden of depressive and anxiety disorders in 204 countries and territories in 2020 due to the COVID19 pandemic. The Lancet. 2021 Nov 6;398(10312):1700-12.

5. Fan FC, Zhang SY, Cheng Y. Incidence of psychological illness after coronavirus outbreak: a meta-analysis study. J Epidemiol Community Health. 2021 Feb 23.

6. Taquet M, Luciano S, Geddes JR, Harrison PJ. Bidirectional associations between COVID19 and psychiatric disorder: retrospective cohort studies of 62354 COVID-19 cases in the USA. The Lancet Psychiatry. 2021 Feb 1;8(2):130-40.

7. Rogers JP, Chesney E, Oliver D, Pollak TA, McGuire P, Fusar-Poli P, Zandi MS, Lewis G, David AS. Psychiatric and neuropsychiatric presentations associated with severe coronavirus infections: a systematic review and meta-analysis with comparison to the COVID-19 pandemic. The Lancet Psychiatry. 2020 Jul 1;7(7):611-27.

8. Kaseda ET, Levine AJ. Post-traumatic stress disorder: a differential diagnostic consideration for COVID-19 survivors. The Clinical Neuropsychologist. 2020 Nov 16;34(7-8):1498-514.

9. Moreno-Pérez O, Merino E, Leon-Ramirez JM, Andres M, Ramos JM, Arenas-Jiménez J, Asensio S, Sanchez R, Ruiz-Torregrosa P, Galan I, Scholz A. Post-acute COVID-19 syndrome. Incidence and risk factors: A Mediterranean cohort study. Journal of Infection. 2021 Mar 1;82(3):378-83.

10. Crook H, Raza S, Nowell J, Young M, Edison P. Long covid-mechanisms, risk factors, and management. bmj. 2021 Jul 26;374. 
medRxiv preprint doi: https://doi.org/10.1101/2022.02.07.22270646; this version posted February 9, 2022. The copyright holder for this preprint (which was not certified by peer review) is the author/funder, who has granted medRxiv a license to display the preprint in perpetuity. It is made available under a CC-BY-NC-ND 4.0 International license .

11. Augustin M, Schommers P, Stecher M, Dewald F, Gieselmann L, Gruell H, Horn C, Vanshylla K, Di Cristanziano V, Osebold L, Roventa M. Post-COVID syndrome in nonhospitalised patients with COVID-19: a longitudinal prospective cohort study. The Lancet Regional Health-Europe. 2021 Jul 1;6:100122.

12. Mendez R, Balanzá - Martínez V, Luperdi SC, Estrada I, Latorre A, González - Jiménez P, Feced L, Bouzas L, Yepez K, Ferrando A, Hervas D. Short - term neuropsychiatric outcomes and quality of life in COVID - 19 survivors. Journal of internal medicine. 2021 Feb 3.

13. Vannorsdall T, Oh ES. Post - acute cognitive and mental health outcomes amongst COVID - 19 survivors: early findings and a call for further investigation. Journal of Internal Medicine. 2021 Sep 1.

14. Groff D, Sun A, Ssentongo AE, Ba DM, Parsons N, Poudel GR, Lekoubou A, Oh JS, Ericson JE, Ssentongo P, Chinchilli VM. Short-term and long-term rates of post-acute sequelae of SARS-CoV-2 infection: a systematic review. JAMA network open. 2021 Oct 1;4(10):e2128568-.

15. Forte G, Favieri F, Tambelli R, Casagrande M. COVID-19 pandemic in the Italian population: validation of a post-traumatic stress disorder questionnaire and prevalence of PTSD symptomatology. International Journal of Environmental Research and Public Health. 2020 Jan;17(11):4151.

16. Khubchandani J, Price JH, Sharma S, Webb FJ. COVID-19 Infection Survivors and the Risk of Depression and Anxiety Symptoms: A Nationwide Study of Adults in the United States. European Journal of Internal Medicine. 2022 Jan 12.

17. Taquet M, Luciano S, Geddes JR, Harrison PJ. Bidirectional associations between COVID19 and psychiatric disorder: retrospective cohort studies of 62354 COVID-19 cases in the USA. The Lancet Psychiatry. 2021 Feb 1;8(2):130-40.

18. Varatharaj A, Thomas N, Ellul MA, Davies NW, Pollak TA, Tenorio EL, Sultan M, Easton A, Breen G, Zandi M, Coles JP. Neurological and neuropsychiatric complications of COVID-19 in 153 patients: a UK-wide surveillance study. The Lancet Psychiatry. 2020 Oct $1 ; 7(10): 875-82$.

19. Pinna P, Grewal P, Hall JP, Tavarez T, Dafer RM, Garg R, Osteraas ND, Pellack DR, Asthana A, Fegan K, Patel V. Neurological manifestations and COVID-19: experiences from a tertiary care center at the frontline. Journal of the Neurological Sciences. 2020 Aug 15;415:116969.

20. Orrù G, Rebecca C, Gemignani A, Conversano C. Psychological intervention measures during the COVID-19 pandemic.

21. Biagianti B, Zito S, Fornoni C, Ginex V, Bellani M, Bressi C, Brambilla P. Developing a Brief Tele-Psychotherapy Model for COVID-19 Patients and Their Family Members.

22. Damiano RF, Di Santi T, Beach S, Pan PM, Lucchetti AL, Smith FA, Forlenza OV, Fricchione GL, Miguel EC, Lucchetti G. Mental health interventions following COVID-19 and other coronavirus infections: a systematic review of current recommendations and metaanalysis of randomized controlled trials. Brazilian Journal of Psychiatry. 2021 Apr 12.

23. Liu K, Chen Y, Wu D, Lin R, Wang Z, Pan L. Effects of progressive muscle relaxation on anxiety and sleep quality in patients with COVID-19. Complementary therapies in clinical practice. 2020 May 1;39:101132.

24. Sotoudeh HG, Alavi SS, Akbari Z, Jannatifard F, Artounian V. The effect of brief crisis intervention package on improving quality of life and mental health in patients with COVID19. Iranian journal of psychiatry. $2020 \mathrm{Jul} ; 15(3): 205$. 
medRxiv preprint doi: https://doi.org/10.1101/2022.02.07.22270646; this version posted February 9, 2022. The copyright holder for this preprint (which was not certified by peer review) is the author/funder, who has granted medRxiv a license to display the preprint in perpetuity. It is made available under a CC-BY-NC-ND 4.0 International license .

25. Islam MN, Islam AN. A systematic review of the digital interventions for fighting COVID19: the Bangladesh perspective. Ieee Access. 2020 Jun 15;8:114078-87.

26. Rauschenberg C, Schick A, Hirjak D, Seidler A, Paetzold I, Apfelbacher C, Riedel-Heller SG, Reininghaus U. Evidence synthesis of digital interventions to mitigate the negative impact of the COVID-19 pandemic on public mental health: rapid meta-review. Journal of medical Internet research. 2021 Mar 10;23(3):e23365.

27. Khilnani A, Schulz J, Robinson L. The COVID-19 pandemic: new concerns and connections between eHealth and digital inequalities. Journal of Information, Communication and Ethics in Society. 2020 Jun 23.

28. Gega L, Aboujaoude E. How Digital Technology Mediated the Effects of the COVID-19 Pandemic on Mental Health: The Good, the Bad, and the Indifferent. Frontiers in Digital Health. 2021;3.

29. Wong AW, Shah AS, Johnston JC, Carlsten C, Ryerson CJ. Patient-reported outcome measures after COVID-19: a prospective cohort study. European Respiratory Journal. 2020 Nov 1;56(5).

30. Schou TM, Joca S, Wegener G, Bay-Richter C. Psychiatric and neuropsychiatric sequelae of COVID-19-A systematic review. Brain, Behavior, and Immunity. 2021 Oct 1;97:328-48.

31. Schwab K, Schwitzer E, Qadir N. Post-Acute Sequelae of COVID-19 Critical Illness. Critical Care Clinics. 2022 Jan 10.

32. Moher D, Shamseer L, Clarke M, Ghersi D, Liberati A, Petticrew M, Shekelle P, Stewart LA. Preferred reporting items for systematic review and meta-analysis protocols (PRISMA-P) 2015 statement. Systematic reviews. 2015 Dec;4(1):1-9.

33. Caliendo AM, Hanson KE. RELATED PATHWAYS. COVID-19 diagnosis. UpToDate. 2022 Jan 21.

34. Griffith GJ, Morris TT, Tudball MJ, Herbert A, Mancano G, Pike L, Sharp GC, Sterne J, Palmer TM, Smith GD, Tilling K. Collider bias undermines our understanding of COVID-19 disease risk and severity. Nature communications. 2020 Nov 12;11(1):1-2.

35. Page MJ, McKenzie JE, Bossuyt PM, Boutron I, Hoffmann TC, Mulrow CD, Shamseer L, Tetzlaff JM, Akl EA, Brennan SE, Chou R. The PRISMA 2020 statement: an updated guideline for reporting systematic reviews. Bmj. 2021 Mar 29;372.

\section{Supplementary material}

\section{Appendix 1 PubMed search strategy (February 07, 2021)}

\begin{tabular}{|c|c|}
\hline \#1 & $\begin{array}{l}\text { (("SARS-CoV-2"[MeSH Terms] OR "COVID-19"[MeSH Terms] OR "COVID-19 } \\
\text { breakthrough infections"[Supplementary Concept] OR "COVID-19 vaccine booster } \\
\text { shot"[Supplementary Concept] OR "post-acute COVID-19 syndrome"[Supplementary } \\
\text { Concept] OR "COVID-19 stress syndrome"[Supplementary Concept] OR "COVID-19 } \\
\text { post-intensive care syndrome"[Supplementary Concept] OR "coronavirus disease } \\
\text { 2019"[Text Word] OR "SARS-CoV-2"[Text Word]) AND "COVID-19"[Text Word]) } \\
\text { OR "Risk Factors"[MeSH Terms] }\end{array}$ \\
\hline$\# 2$ & $\begin{array}{l}\text { "Mental Disorders"[MeSH Terms] OR "Diagnostic and Statistical Manual of Mental } \\
\text { Disorders"[MeSH Terms] OR "Substance-Related Disorders"[MeSH Terms] OR } \\
\text { "Neurocognitive Disorders"[MeSH Terms] OR "Patient Health Questionnaire"[MeSH } \\
\text { Terms] OR "depress*"[Text Word] OR "anxi*"[Text Word] OR "substance use }\end{array}$ \\
\hline
\end{tabular}


medRxiv preprint doi: https://doi.org/10.1101/2022.02.07.22270646; this version posted February 9, 2022. The copyright holder for this preprint (which was not certified by peer review) is the author/funder, who has granted medRxiv a license to display the preprint in perpetuity.

It is made available under a CC-BY-NC-ND 4.0 International license.

\begin{tabular}{|l|l|}
\hline & $\begin{array}{l}\text { disorder"[Text Word] OR "posttraumatic stress disorder"[Text Word] OR } \\
\text { "psycho*"[Text Word] OR "self-harm"[Text Word] OR "suicide"[Text Word] }\end{array}$ \\
\hline$\# 3$ & $\begin{array}{l}\text { "Causality"[MeSH Terms] OR "etiology"[MeSH Subheading] OR "Causality"[Text } \\
\text { Word] OR "etiology"[Text Word] }\end{array}$ \\
\hline$\# 4$ & $\# 1$ AND \#2 AND \#3 \\
\hline
\end{tabular}

